\title{
Business Engineering Model
}

\author{
Hubert Österle, Dieter Blessing
}

Business Engineering bedeutet systematische Entwicklung neuer Geschäftslösungen. Business Engineering zerlegt die Transformation von Unternehmen in beherrschbare Schritte, gibt Anleitung zur Bearbeitung dieser Schritte und verbindet diese in Vorgehensmodellen für Projekte. Der Aufsatz erklärt die Arbeitsweise des Business Engineering anhand eines durchgängigen Beispiels und beschreibt die Grundprinzipien dieser neuen Disziplin.

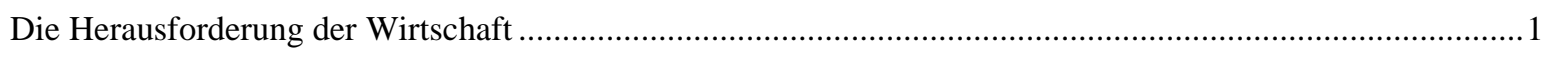

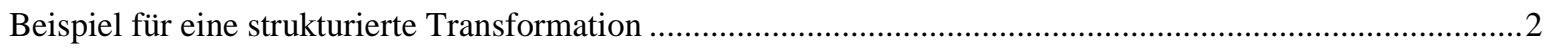

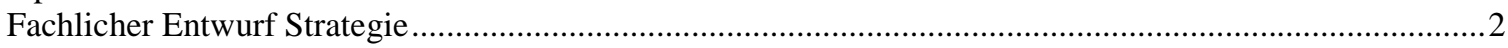

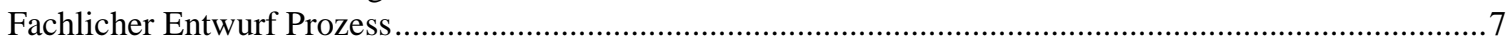

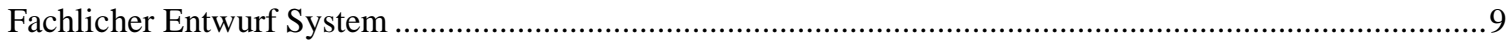

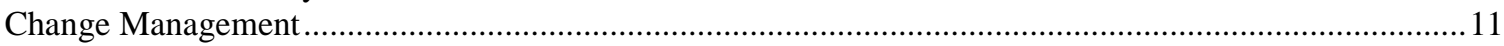

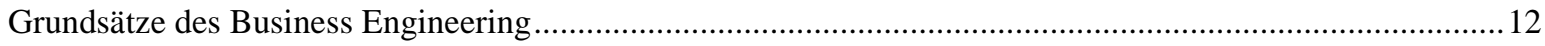

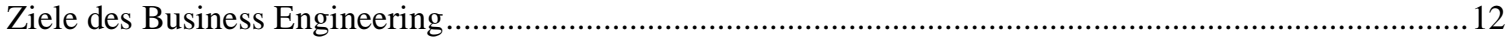

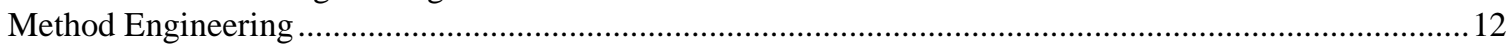

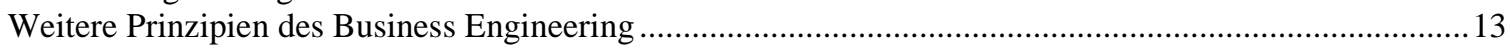

Methoden und Werkzeuge für das Business Engineering ............................................................. 14

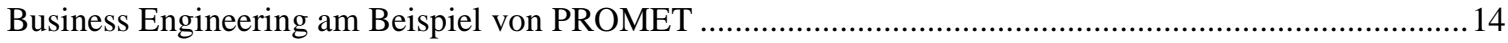

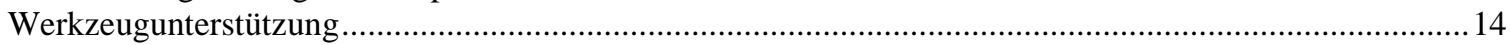

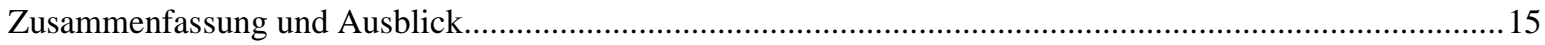

\section{Die Herausforderung der Wirtschaft}

Die Transformation vom Industrie- ins Informationszeitalter ist eine gigantische Herausforderung für Wirtschaft und Gesellschaft. Viele tausend Projekte pro Jahr bringen neue oder verbesserte Geschäftslösungen hervor. Mittelständische Unternehmen mit 1000 Mitarbeitern betreiben fünf bis 15 Projekte parallel, Grossunternehmen können durchaus auf 500 Projekte und mehr kommen.

Nicht alle Projekte sind erfolgreich. Erfolgsgeschichten wechseln sich ab mit Nachrichten über gescheiterte Projekte. Laut einer Untersuchung von über 600 IT-Vorhaben durch Cambridge Technology Partners funktionieren $95 \%$ der Projekte nicht zufriedenstellend [vgl. Aschwanden 1998].

Viele Unternehmen sind dabei, ihre administrativen Prozesse auf der Basis von Enterprise Resource Planning Paketen oder ihre Verkaufsprozesse mittels Software für das Customer Relationship Management zu reorganisieren. Während einige, vor allem grosse Unternehmen derartige Projekte nach Aufwendungen von vielen Millionen Euro stoppen oder neu aufsetzen, berichten andere von Erfolgen mit kurzen Einführungszeiten und beherrschbaren Projektkosten.

Was macht ein Projekt zum Erfolg? Projektmanagement, Know-how, Technologie, Change Management? Die Beschränkung des Projektumfanges, die Zerlegung in überschaubare Projektabschnitte und ein systematisches Vorgehen, das die Einzelergebnisse verbindet, erhöhen die Erfolgsaussichten von Projekten deutlich [vgl. Dolmetsch et al. 1998].

Business Engineering hat das Ziel, die Erkenntnisse über die Transformation aus Wissenschaft und Praxis zusammenzufassen und daraus Konzepte und Instrumente zur Planung und Realisierung von Geschäftslösungen des Informationszeitalters abzuleiten. Es bringt betriebswirtschaftliches und informationstechnisches Wissen zusammen und verbindet es mit allen Aspekten der Transformation, von Darstellungsmitteln über Vorgehensmodelle bis hin zu kulturellen und politischen Gesichtspunkten.

Das hier vorgestellte Business Engineering Modell soll einen Denkrahmen liefern und dem Business Engineer helfen, einzelne Projekte und Projektaktivitäten im Gesamtzusammenhang zu verstehen. 


\section{Beispiel für eine strukturierte Transformation}

Business Engineering strukturiert die Transformation. Es unterteilt Transformationsvorhaben in die Entwicklung von Strategie, Prozess und System, zerlegt diese Ebenen in beherrschbare, kleine Schritte und verbindet sie über die Ergebnisse. Business Engineering unterstützt dabei nicht nur die fachliche Gestaltung, sondern auch das Change Management. Im folgenden stellen wir an einem stark vereinfachten Projektbeispiel den Ansatz des Business Engineerings dar.

Ein multinationaler Konzern der Unterhaltungselektronik, die UNTEL AG, verkauft bis heute vorwiegend Stereoanlagen, DVD-Player, Spiele, CDs und ähnliche Produkte (vgl. z. B. für Sony [Kunii 1999]). Die Digitalisierung und das Internet verändern die Spielregeln dieser Branche grundlegend. Die UNTEL startet daher verschiedene Projekte, in denen sie die neuen Formen des Geschäftes erproben und aufbauen will.

Sie gründet das Tochterunternehmen Intertainment.com, das sich ausschliesslich der musikalischen Unterhaltung über das Internet widmet (vgl. z. B. cdnow.com, launch.com, mp3.com, sdmi.org). Im Projekt „Music-for-kids“ entstehen folgende Dokumente (vgl. Abb. 1) zur Planung und Umsetzung des neuen Geschäftes (stark vereinfacht).
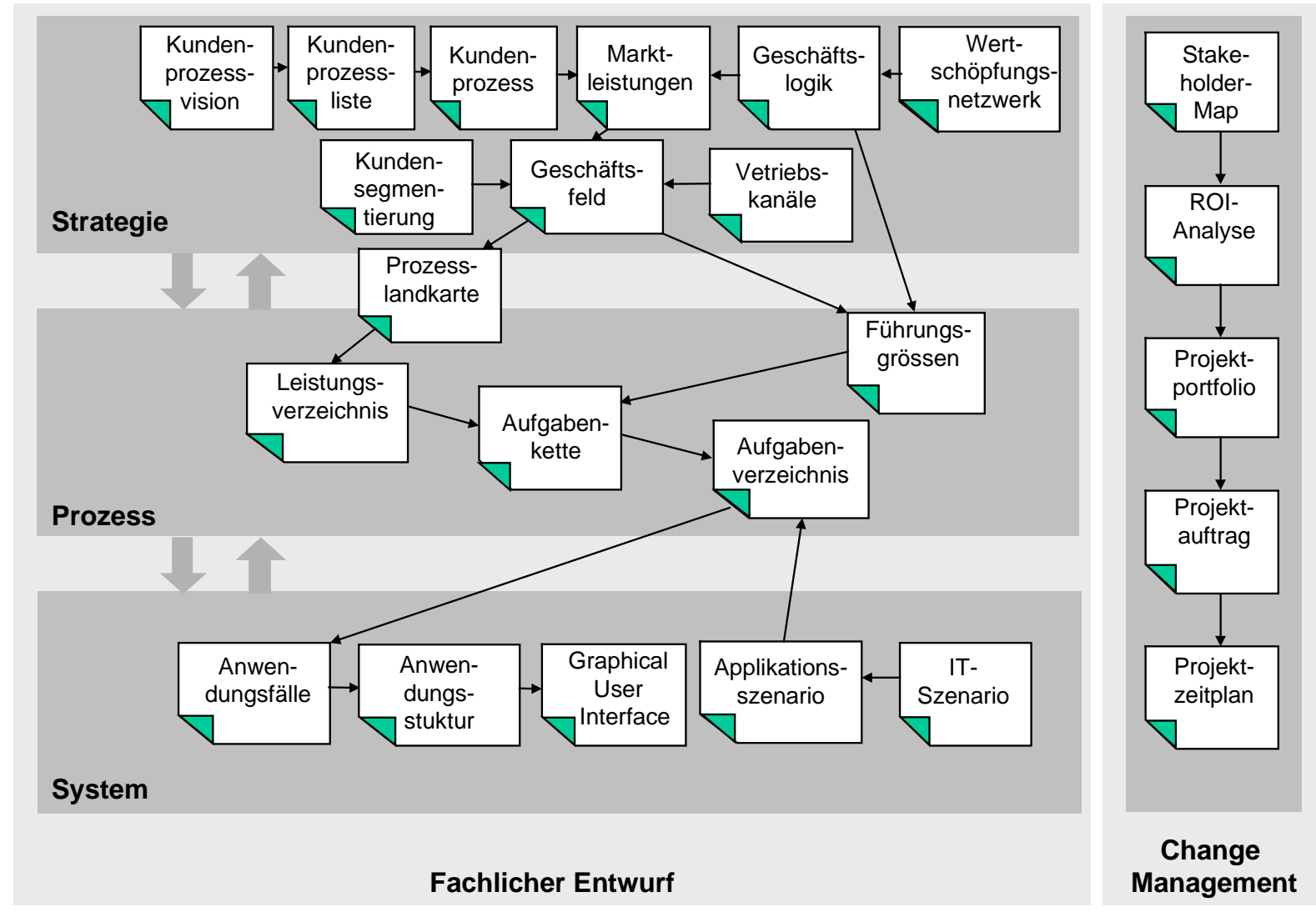

Abb. 1. Zerlegung der Transformation im Business Engineering

\section{Fachlicher Entwurf Strategie}

Das Projekt geht vom Kunden und seinem Prozess der Musikunterhaltung aus. Das Dokument „Kundenprozessvision“ (vgl. Abb. 2) fasst die Vorstellungen des Projektteams zusammen, welche dieses über den idealen Kundenprozess entwickelt hat. 


\section{Zweck}

Der Kunde soll Musikunterhaltung nach seinem Geschmack in beliebigen Situationen und mit grösstmöglichem Komfort erhalten. Situationen sind beispielsweise das Autofahren, die Erholung am Ende des Arbeitstages und der Konzertbesuch.

\section{Zielgruppe}

Intertainment.com richtet sich zunächst an Jugendliche zwischen 12 und 18, die mit den digitalen Medien vertraut sind.

\section{Leistungen}

- Bestellservice

- Verkauf von CDs, Videos, Konzertkarten und Fanartikeln, wie T-Shirts, Bücher, Fotos usw.

- Abspielen von Ausschnitten von Songs und Videos vor dem Kauf

Online-Abspielen von Musik

Die Musik wird mit einem digitalen Wasserzeichen personalisiert und lässt sich nur auf einer

Playersoftware abspielen, die die Daten des Käufers kodiert enthält.

- Weiterbearbeitung von Songs

...

- $\quad$ Erstellen individueller Audio-CDs

...

- $\quad$ Informationen über die Musikszene

...

- Virtuelle Gemeinschaft

$\ldots$

Abb. 2. Kundenprozessvision Musikunterhaltung

Die Bedürfnisse der Kunden sind je nach Kundensegment unterschiedlich, beispielsweise abhängig vom Einkommen. Daher spezifiziert die Intertainment.com ihre Kundensegmente anhand verschiedener Deskriptoren (vgl. Abb. 3).

\begin{tabular}{|l|l|l|l|}
\hline \multicolumn{1}{|c|}{ Kundensegment } & Kids & Junge Erwachsene & $\ldots$ \\
\hline Deskriptor & & & \\
\hline Alter & $12-16$ & $16-20$ & \\
\hline Familienstand & ledig & ledig & \\
\hline Raushaltseinkommen & 100 bis 200 CHF / Monat & 200 bis 500 CHF / Monat & \\
\hline Persönlichkeitsstruktur & Industrieländer & Darstellung des Erwachsenseins & Industrieländer \\
\hline$\ldots$ & $\ldots$ & $\ldots$ & \\
\hline
\end{tabular}

Abb. 3. Kundensegmentierung

Vor dem Hintergrund der Kundenprozessvision und der Kundensegmente sammelt das Projektteam die Situationen, in denen der Kunde Musikunterhaltung wünscht (vgl. Abb. 4).

\begin{tabular}{|l|l|}
\hline Bezeichnung & Beschreibung \\
\hline Bewusstes Musikhören & $\begin{array}{l}\text { Der Kunde hört bewusst Musik seiner gewünschten Stilrichtung. Er liest } \\
\text { Informationen zur Musik und nimmt an einem elektronischen Diskussionsforum } \\
\text { teil. }\end{array}$ \\
\hline Konzertbesuch & $\begin{array}{l}\text { Der Kunde besucht ein Konzert einer bestimmten Stilrichtung (Rock, Klassik, } \\
\text { Jazz usw.). }\end{array}$ \\
\hline$\ldots$ & $\ldots$ \\
\hline
\end{tabular}

Abb. 4. Kundenprozessliste 
In jeder dieser Situationen läuft beim Kunden ein anderer (Teil-)Prozess der Musikunterhaltung ab (vgl. Abb. 5).

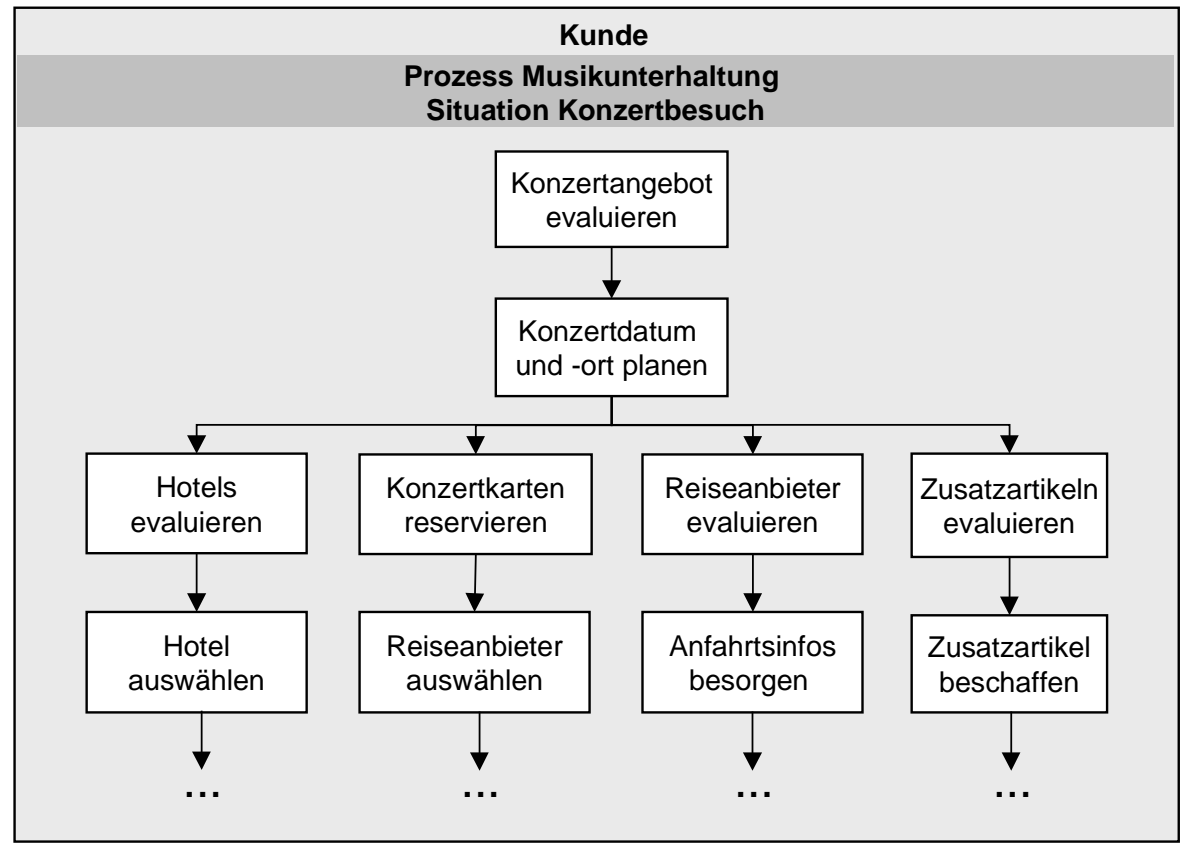

Abb. 5. Kundenprozess Musikunterhaltung (Situation Konzertbesuch)

Das Verständnis des Prozesses des Kunden ist Voraussetzung für das Verständnis von dessen Bedürfnissen, Problemen und seiner Wahrnehmung von Qualität. Da der geplante Kundenprozess bis heute nicht existiert und somit nicht beobachtet werden kann, ist die graphische Darstellung des Kundenprozesses ein Hilfsmittel, diesen Prozess vorstellbar und kommunizierbar zu machen.

Das Ziel der Intertainment.com ist es, den Kundenprozess umfassend aus einer Hand anzubieten. Die Intertainment.com muss daher Produkte und Dienstleistungen auch von anderen Anbietern in ihr Angebot aufnehmen. Das Wertschöpfungsnetzwerk (Supply Chain) verschafft einen Überblick über die Marktteilnehmer und ihrer Leistungen zur Befriedigung des Kundenbedürfnisses (vgl. Abb. 6). Die Intertainment.com gewinnt damit auch einen Überblick über ihre Position im Markt, insbesondere mögliche Konkurrenten und Partner.

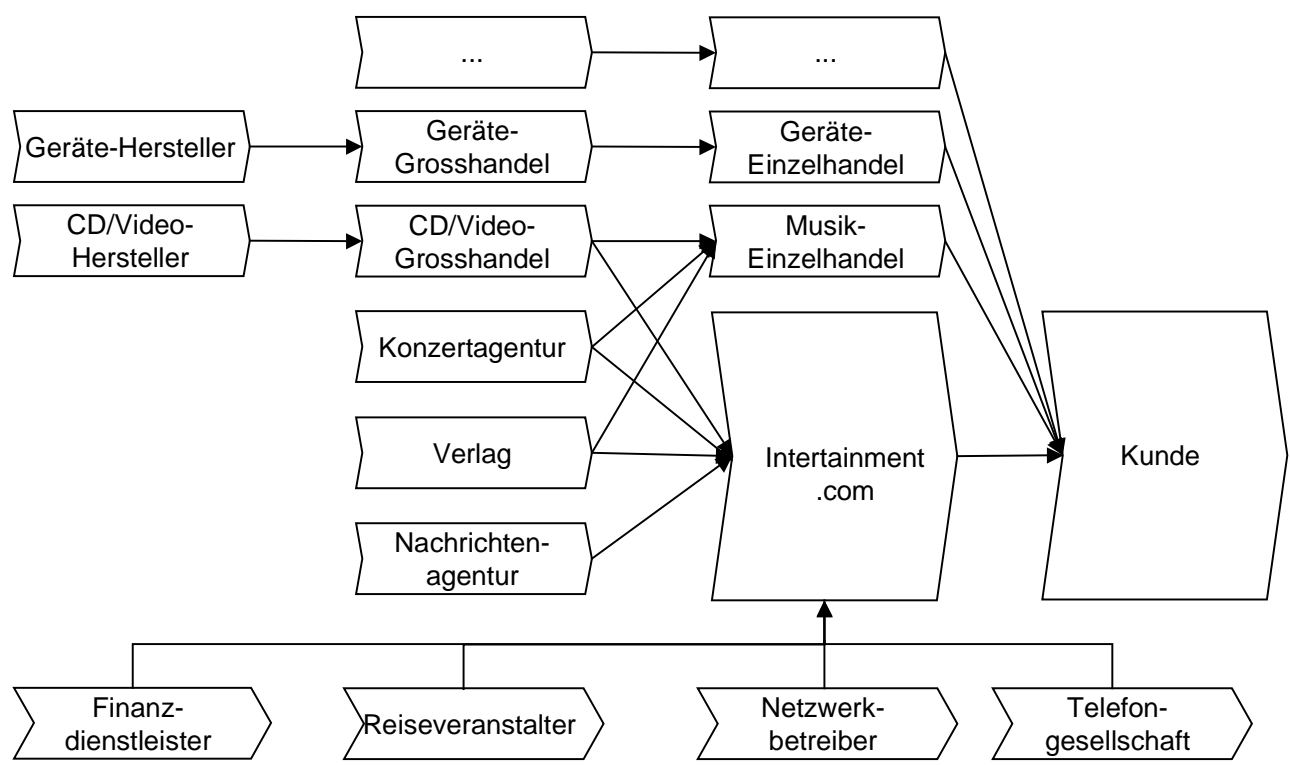

Abb. 6. Wertschöpfungsnetzwerk 
Bevor die Strategieentwicklung in die Detailplanung, vor allem in die finanzielle Planung, einsteigt, muss die Logik des Geschäftes klar sein. Ein Wirkungsnetzwerk [vgl. Gomez/Probst 1997] ist ein einfaches Hilfsmittel, um die Mechanismen des Marktes verstehen zu lernen. Das Beispiel der Intertainment.com verdeutlicht die Regeln des Internetzeitalters: Marktmacht ist Voraussetzung für den Zugriff auf Kunden und die Anbindung von Lieferanten. Je mehr Kunden und je mehr Lieferanten, desto grösser die Marktmacht. Diese wiederum senkt die Beschaffungskosten und die Kosten pro Transaktion und verbessert damit wieder die Position im Markt (vgl. Abb. 7).

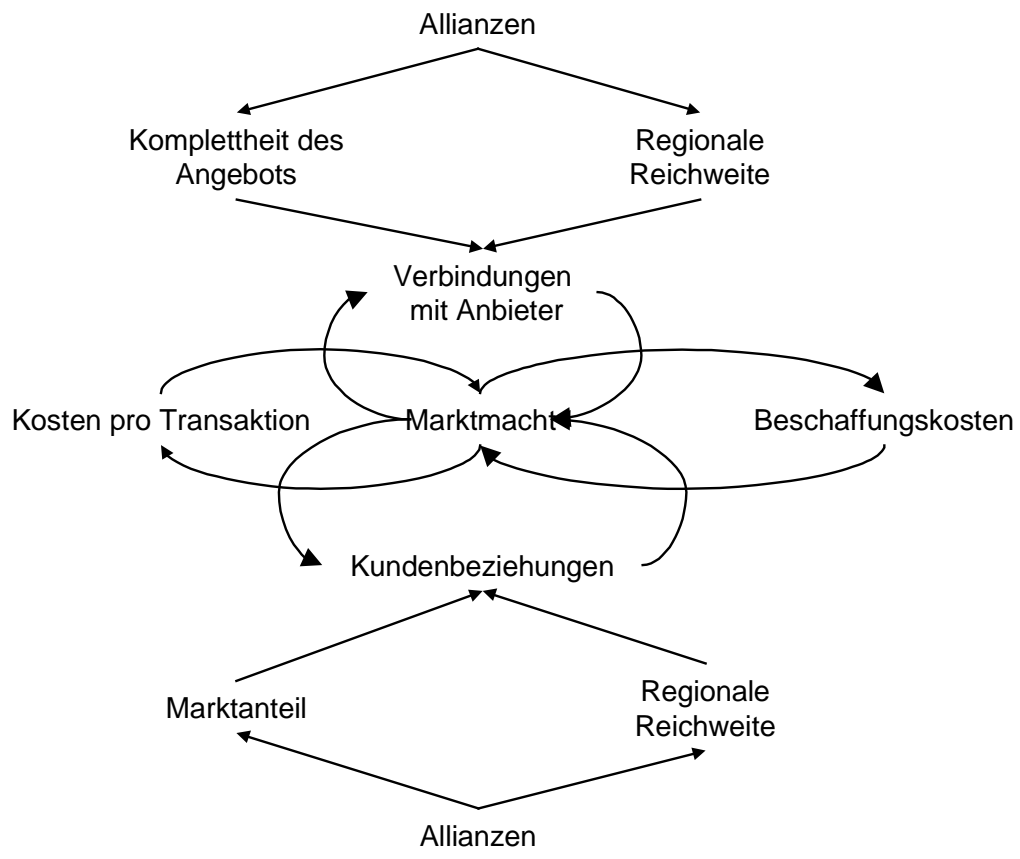

Abb. 7. Geschäftslogik

Aus den Bedürfnissen des Kundenprozesses und aus der Situation im Wertschöpfungsnetzwerk sind die Marktleistungen der Intertainment.com abzuleiten (vgl. Abb. 8).

\begin{tabular}{|l|l|l|}
\hline Marktleistung & Beschreibung & Preis \\
\hline Informationsservice & $\begin{array}{l}\text { Aktuelle Informationen zur Musikszene in Kooperation mit } \\
\text { einer Musikzeitschrift, als Push- und Pull-Service }\end{array}$ & gratis \\
\hline Veranstaltungsservice & $\begin{array}{l}\text { Reservation und Bereitstellung von Eintrittskarten, } \\
\text { Buchung und Abrechnung von Hotels, .. }\end{array}$ & $5 \%$ Provision \\
\hline Geräteverkauf & $\begin{array}{l}\text { Online-Shop für das gesamte Gerätesortiment der UNTEL } \\
\text { auf dem Gebiet der Unterhaltungselektronik }\end{array}$ & $\begin{array}{l}10 \% \text { unter } \\
\text { Einzelhandel }\end{array}$ \\
\hline$\ldots$ & $\ldots$ & $\ldots$ \\
\hline
\end{tabular}

Abb. 8. Marktleistungen

Da die Intertainment.com ausschliesslich über ihr ProzessPortal auf dem Internet verkaufen will, ist in diesem Fall eine Aufstellung möglicher Vertriebskanäle nicht notwendig.

Auf Basis der bisherigen Ergebnisse (Dokumente) lassen sich nun die Geschäftsfelder der Intertainment.com ableiten. Ein Geschäftsfeld ist eine Kombination von Marktleistungen, Kundensegmenten und Vertriebskanälen, das homogen geführt werden kann, für das also gemeinsame Marketingaktionen, dieselben Produktmanager, die gleiche Abwicklung, die gleiche Erfolgsmessung usw. möglich sind. Die Intertainment.com verzichtet auf eine Differenzierung der Geschäftsfelder nach Kundensegmenten wie, z.B. Kids, richtet aber für die Marktleistungen für den Kundenprozess Konzertbesuch ein separates Geschäftsfeld ein (vgl. Abb. 9). 


\begin{tabular}{|c|c|c|c|c|c|c|}
\hline \multicolumn{7}{|c|}{ Abgrenzung des strategischen Geschäftsfeldes } \\
\hline $\begin{array}{ll}\text { Produktgruppe(n) } \\
\end{array}$ & \multicolumn{6}{|c|}{ Konzertbesuch } \\
\hline Kundengruppe / Region & \multicolumn{6}{|l|}{ alle } \\
\hline Vertriebskanal & \multicolumn{6}{|l|}{ ProzessPortal } \\
\hline \multicolumn{7}{|l|}{ Kurz-Charakteristika } \\
\hline \multicolumn{7}{|c|}{$\begin{array}{l}\text { Ziel dieses Geschäftsfeldes ist es, Konzert- und Reiseangebote den spezifischen finanziellen Möglichkeiten } \\
\text { der Kundensegmente entsprechend bereitzustellen. Der Einstieg in dieses Geschäft gelingt durch die } \\
\text { Übernahme einer Musikzeitschrift, die diesen Service bisher postalisch angeboten hat, relativ schnell. }\end{array}$} \\
\hline \multicolumn{7}{|c|}{$\begin{array}{l}\text { Spezialisten aus dem Tourismus und der Konzertveranstaltung betreiben dieses Geschäftsfeld autonom. Sie } \\
\text { finanzieren die Gratis-Dienstleistungen (z. B. Information) ausserhalb ihres Geschäftsfeldes als Umsatzmittler } \\
\text { mit. }\end{array}$} \\
\hline \multicolumn{7}{|c|}{ Quantitative Entwicklung (reale Grössen) } \\
\hline Entwicklungsgrössen & \begin{tabular}{l|l|} 
& 2000 \\
\end{tabular} & 2001 & 2002 & 2003 & 2004 & 2005 \\
\hline Gesamtmarkt total (Mio.) & 240 & 245 & 250 & 257 & 265 & 273 \\
\hline Marktwachstum in \% & 2 & 2 & 2 & 3 & 3 & 3 \\
\hline Nettoerlös Intertainment (Mio.) & 72 & 75 & 85 & 95 & 108 & 125 \\
\hline Marktanteil in \% & 30 & 31 & 34 & 37 & 41 & 46 \\
\hline Deckungsbeitrag 1 total (Mio.) & 19 & 18 & 18 & 19 & 19 & 20 \\
\hline Cashflow total (Mio.) & -1 & 9 & 11 & 14 & 17 & 20 \\
\hline
\end{tabular}

Abb. 9. Geschäftsfeld Konzertbesuch

Als letztes Dokument der Strategieentwicklung entsteht die Prozesslandkarte, eine Darstellung des Kundenprozesses und seiner Teilprozesse, der internen Prozesse der Intertainment.com sowie der wichtigsten Zulieferanten (vgl. Abb. 10). Dies ist beispielsweise der Logistikprozess, den die Intertainment.com vom Mutterhaus, der UNTEL AG, bezieht. 


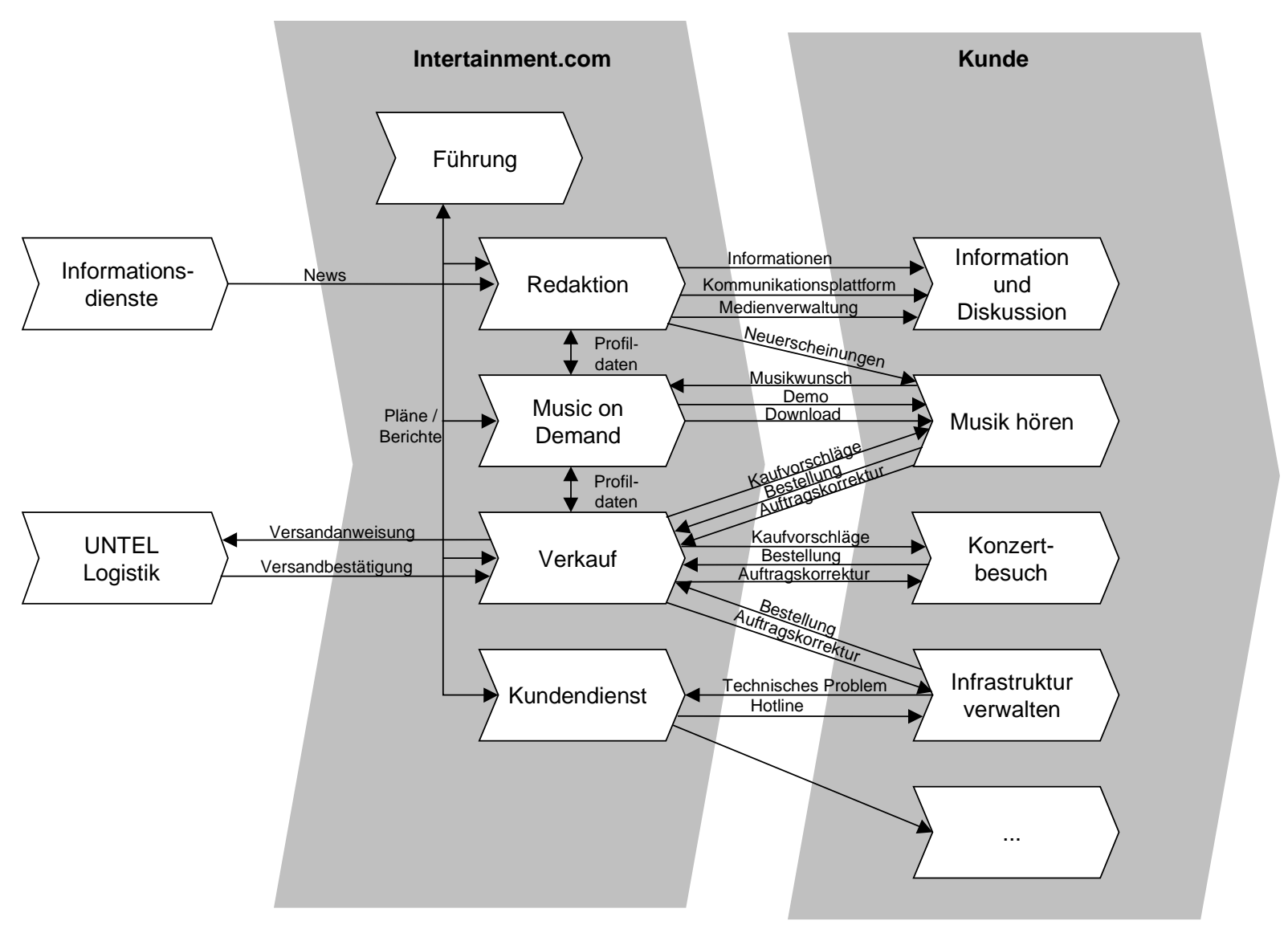

Abb. 10. Prozessarchitektur

\section{Fachlicher Entwurf Prozess}

Jeder der identifizierten Prozesse ist auf dieser Ebene im Detail zu entwerfen. Wir stellen im folgenden den Prozess Verkauf dar.

Da der Kunde in allen Teilprozessen ausser Information und Diskussion Leistungen von der Intertainment.com gegen Bezahlung bezieht, benutzt er den Verkaufsprozess in verschiedenen Situationen. Grundlage für die Entwicklung jedes Prozesses sind die Leistungen, die der Kunde in diesen Situationen benötigt (vgl. Abb. 11).

\begin{tabular}{|l|l|}
\hline Leistung & Beschreibung \\
\hline Kaufvorschläge & $\begin{array}{l}\text { Individualisierte Kaufvorschläge aufgrund des Profils des Käufers } \\
\text { (Interessensgebiete, bisherige Käufe usw.), bei Wunsch Zustellung von } \\
\text { Kaufvorschlägen in Form eines Push-Dienstes, z. B. per eMail }\end{array}$ \\
\hline Bestellung & Bestellung des Käufers im Online-Shop \\
\hline Auftragskorrektur & Korrektur der Bestellung im Online-Shop, in Problemfällen per Telefon \\
\hline Versandanweisung & Freigabe eines Auftrages zur Auslieferung \\
\hline Versandbestätigung & Bestätigung der Auslieferung eines Auftrags \\
\hline Profildaten & $\begin{array}{l}\text { Daten über Interessensgebiete und Verhalten des Kunden, das sich sowohl aus } \\
\text { dessen Informationsbedürfnissen als auch aus dem Kaufverhalten ableitet }\end{array}$ \\
\hline Pläne/Berichte & Pläne und Berichte zu den Führungsgrössen des Prozesses \\
\hline$\ldots$ & $\ldots$ \\
\hline
\end{tabular}


Die Prozessleistungen detaillieren einerseits die Marktleistungen, die in der Strategie bestimmt worden sind, und betrachten zusätzlich die Leistungen an andere Prozesse als den Kundenprozess (z.B. die UNTEL Logistik). Das Leistungsverzeichnis geht vom Prozess aus.

Nun sind die Aufgaben (Aktivitäten) des Prozesses zu formulieren, welche die vorgegebenen Leistungen erzeugen. Wir beginnen mit dem Aufgabenkettendiagramm (vgl. Abb. 12). Geht ein ProzessPortal konsequent vom Kundenprozess aus, so gibt es keinen eigenen Kaufprozess, sondern die Aufgaben des Kaufes sind Bestandteil beinahe jeder Situation im Kundenprozess. Planen wir den Prozess Verkauf auf der Seite der Intertainment.com, müssen wir aber die Aufgaben des Kaufes beim Kunden in einen Prozessausschnitt zusammenfassen.

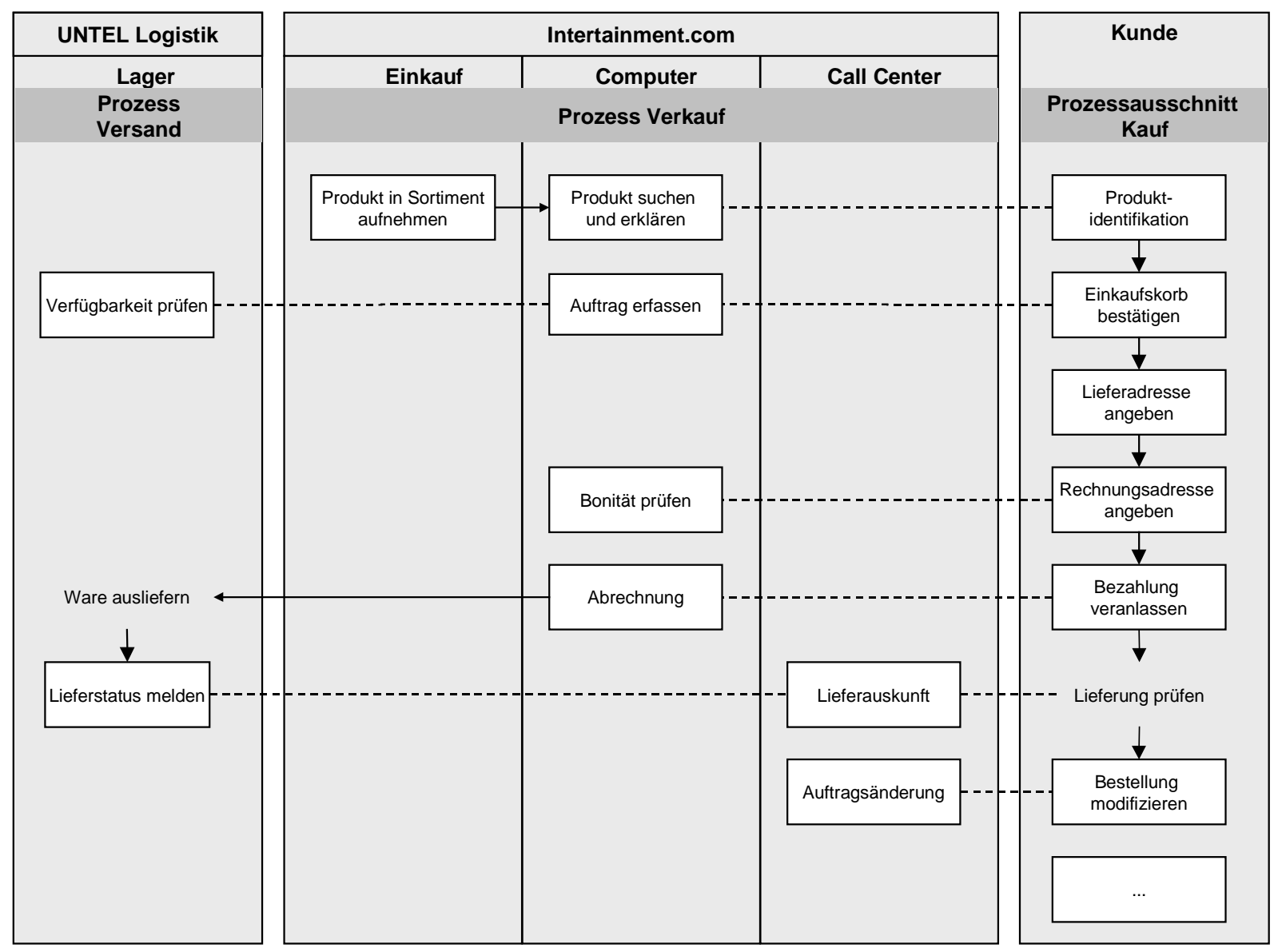

Abb. 12. Aufgabenkettendigramm Prozess Verkauf

Das Aufgabenverzeichnis enthält Angaben zu jeder Aufgabe, ergänzend zum Aufgabenkettendiagramm (vgl. Abb. 13). Diese sind vor allem zur Abschätzung des Personalaufwandes sowie zur Ermittlung der betroffenen Applikationen notwendig.

\begin{tabular}{|l|l|l|l|l|c|}
\hline Aufgabe & $\begin{array}{l}\text { Organisations- } \\
\text { einheit }\end{array}$ & $\begin{array}{l}\text { Applikation / } \\
\text { Datenbasis }\end{array}$ & $\begin{array}{l}\text { Häufigkeit } \\
\text { pro Jahr }\end{array}$ & $\begin{array}{l}\text { Zeitbedarf } / \\
\text { Transaktion } \\
\text { in h }\end{array}$ & $\begin{array}{l}\text { Aufwand } \\
\text { p. a. in h }\end{array}$ \\
\hline $\begin{array}{l}\text { Produkt in Sortiment } \\
\text { aufnehmen }\end{array}$ & Einkauf & Produktkatalog & $5 ' 800$ & 1,25 & $77^{\prime} 250$ \\
\hline $\begin{array}{l}\text { Produkt suchen und } \\
\text { erklären }\end{array}$ & Computer & Produktkatalog & 2 Mio & vollständig computergestützt \\
\hline Auftrag erfassen & Computer & Auftragsabwicklung & $200^{\prime} 000$ & vollständig computergestützt \\
\hline Auftragsänderung & Call Center & Auftragsabwicklung & $63^{\prime} 000$ & 0,1 & 6 \\
\hline$\ldots$ & $\ldots$ & $\ldots$ & $\ldots$ & $\ldots$ & \\
\hline
\end{tabular}


Das Wirkungsnetzwerk beschreibt die Faktoren, die den Geschäftserfolg bestimmen. Das Prozessmanagement muss versuchen, die Vorgaben aus der Strategie auf der Prozessebene umzusetzen und permanent zu verbessern (vgl. Abb. 14).

\begin{tabular}{|l|l|l|}
\hline Kritischer Erfolgsfaktor & Führungsgrösse & $\begin{array}{l}\text { Berechnung bei der Intertainment.com im } \\
\text { Prozess Verkauf }\end{array}$ \\
\hline \multirow{3}{*}{ Kundenbeziehungen } & Neukunden & Anzahl der Neukunden pro Monat \\
\cline { 2 - 3 } & Web-Zugriffe & Anzahl der Zugriffe auf Homepage pro Monat \\
\cline { 2 - 3 } & Prospects & Anzahl der neuen Adressen \\
\cline { 2 - 3 } & Kundenverlust & Anzahl der inaktiven Kunden \\
\hline \multirow{3}{*}{ Beschaffungskosten } & Volumen pro Lieferant & kumuliertes Bestellvolumen \\
\cline { 2 - 3 } & Rabattsatz & Volumensrabatt pro Lieferant \\
\cline { 2 - 3 } & $\ldots$ & \\
\hline$\ldots$ & $\ldots$ & $\ldots$ \\
\hline
\end{tabular}

Abb. 14. Führungsgrössen Prozess Verkauf

\section{Fachlicher Entwurf System}

Das Applikationsszenario zeigt die Applikationen, auf welche der Prozess Verkauf aufbauen kann, in ihrem Zusammenhang (vgl. Abb. 15).

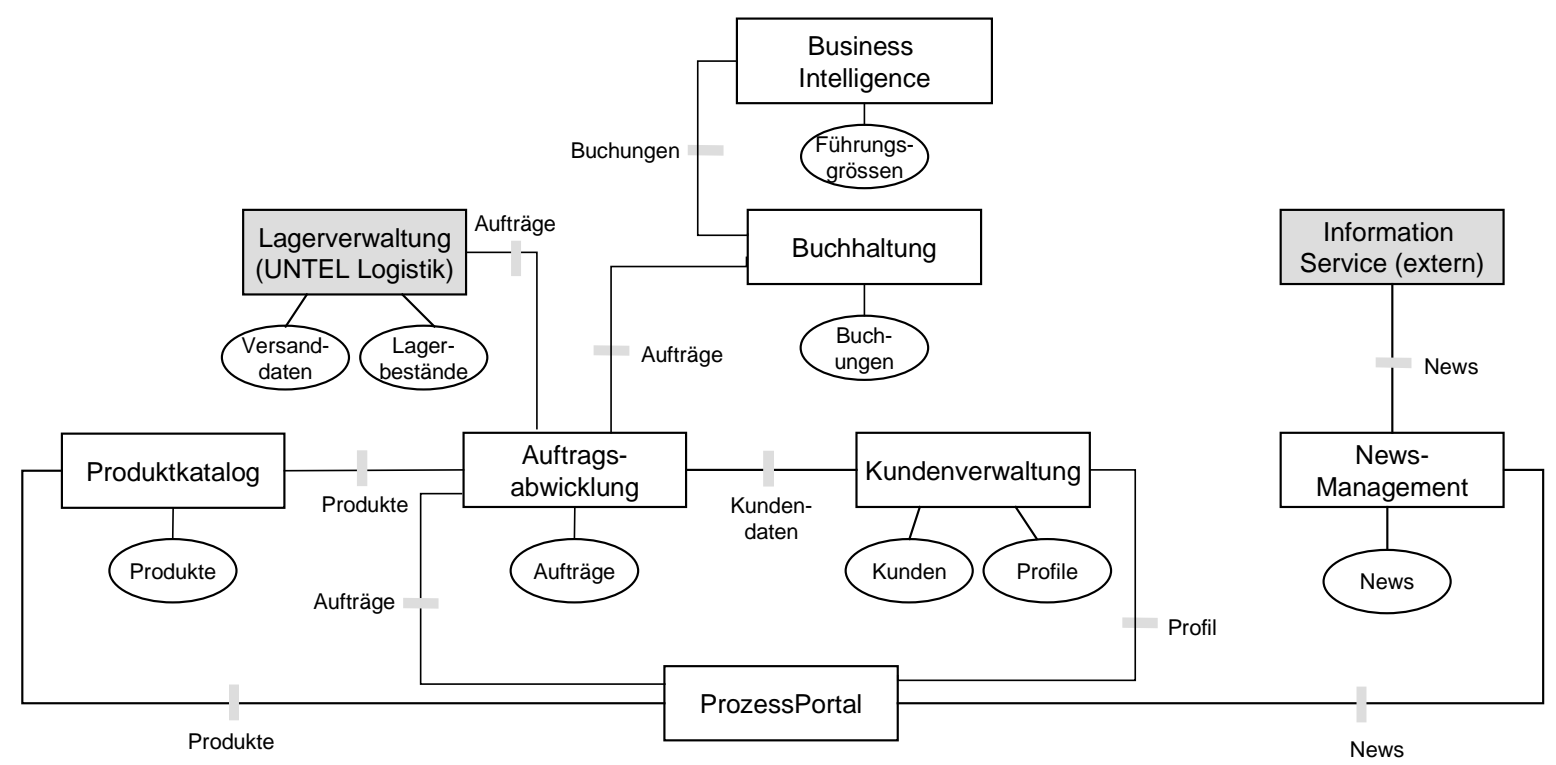

Abb. 15. Applikationsszenario

Ergänzend zum Überblick über die Applikationen benötigen wir eine Sicht auf die zugrundeliegende Informationstechnik in Form von Basissoftware (vgl. Abb. 16). 


\begin{tabular}{|l|l|l|l|}
\hline Organisationseinheit & Komponente & Typ & Produkt \\
\hline Auftragsabwicklung/ZH & $\begin{array}{l}\text { Client- } \\
\text { Betriebssystem } \\
\text { Web-Client }\end{array}$ & Applikation & Windows NT Workstation 4.0 \\
& Applikation & Mircosoft Internet Explorer 5 \\
\hline Informatik/ZH & Web-Server & Applikation & Microsoft Internet Information Server 4.0 \\
& Mail-Server & Applikation & Lotus Notes 5.0 \\
& Index Server & Middleware & Microsoft Index Server \\
& Catalog Server & Middleware & Netscape Compass Server \\
& $\ldots$ & $\ldots$ & $\ldots$ \\
\hline
\end{tabular}

Abb. 16. IT-Szenario

Das Applikations- und IT-Szenario sind eine wichtige Grundlage für die Prozessentwicklung. Sie helfen, die Kosten unterschiedlicher Prozessvarianten frühzeitig abzuschätzen. Die beiden Szenarien gehören zwar zur System-Ebene, werden aber für den Prozessentwurf gebraucht. Die Ebenen des Business Engineering implizieren keine ausschliessliche Top-down-Vorgehensweise.

Aus den Aufgaben, die wir im Prozessentwurf bestimmt haben, leiten wir Anwendungsfälle ab (vgl. Abb. 17). Diese beschreiben die Anforderungen an die Applikationen im Detail.

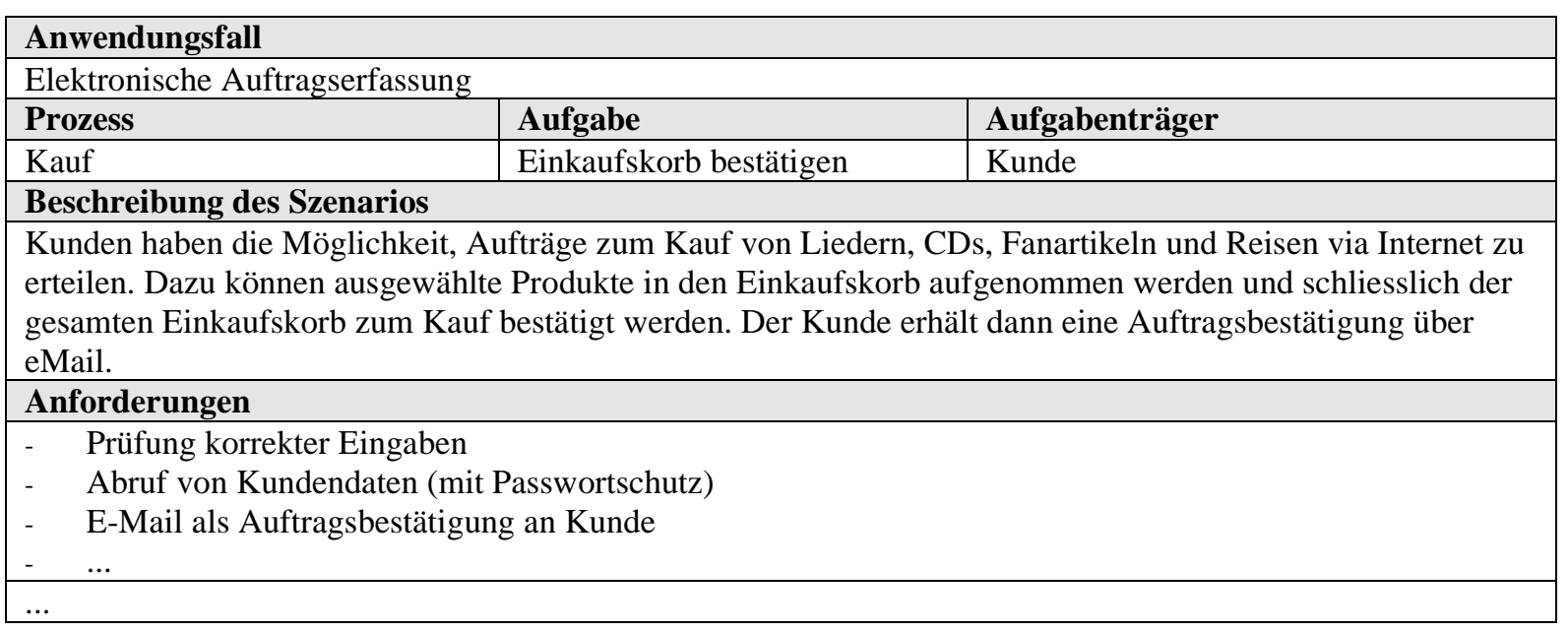

Abb. 17. Anwendungsfälle

Die Abfolge der Bildteile in der graphischen Benutzerschnittstelle beschreibt die dynamische Anwendungsstruktur (vgl. Abb. 18), auf das Dokument Graphical User Interface und weitere Ergebnisse des Systementwurfs verzichten wir hier aus Platzgründen.

\section{Anwendungssfall: Elektronische Auftragserfassung}

\author{
Artikel aufnehmen \\ Fortfahren mit Bestellung \\ Artikel hinzufügen \\ Bestätigung Einkaufskorb
}

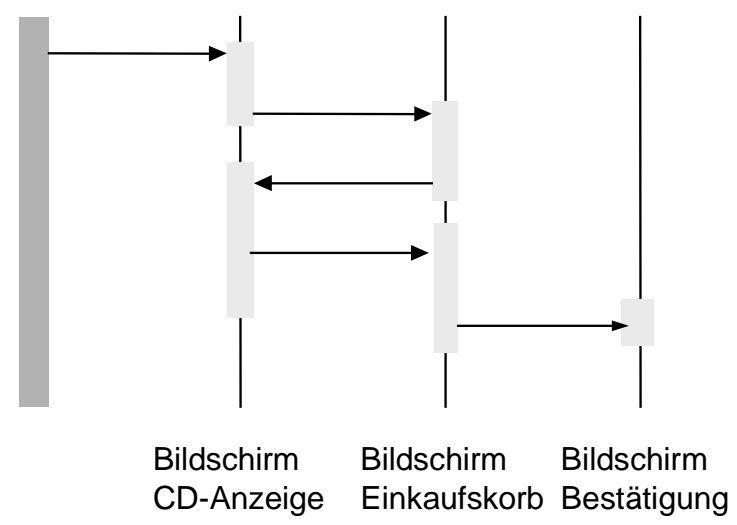




\section{Change Management}

Bis jetzt haben wir die fachliche Lösung des Projektes Intertainment.com behandelt. Das Business Engineering betrachtet jedoch nicht nur diese, sondern mit derselben Wichtigkeit auch das Management der Veränderung. Es beschäftigt sich u. a. mit den Anspruchsgruppen (Stakeholder-Map), mit der Wirtschaftlichkeit der Lösung (ROI-Analyse) und mit dem Projektmanagement. Aus Platzgründen sind hier nur eine Stakeholder-Map (vgl. Abb. 19) und ein Projektzeitplan (vgl. Abb. 20) aufgenommen.

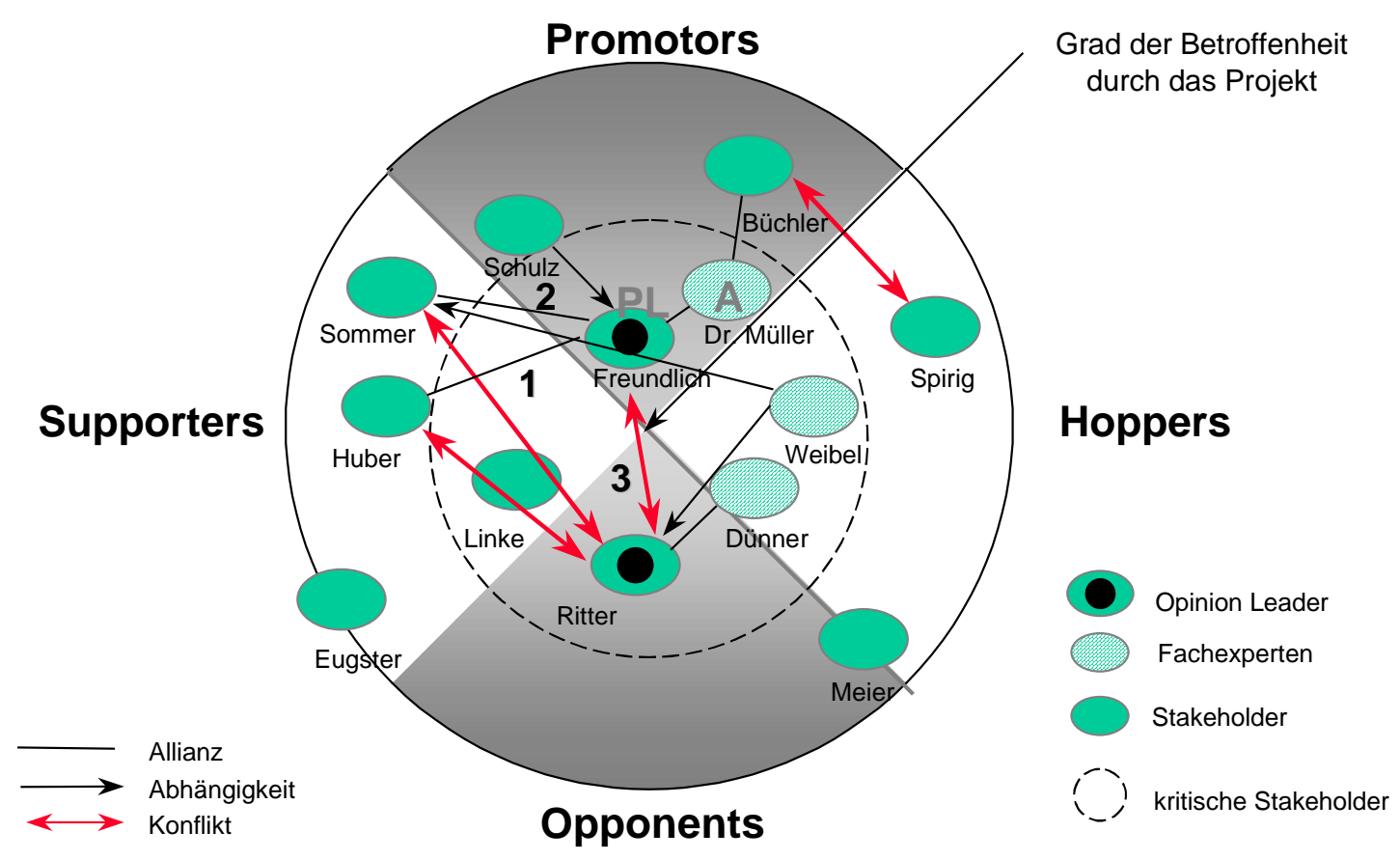

Abb. 19. Stakeholder Map

\begin{tabular}{|c|c|c|c|c|c|c|c|c|c|c|c|c|c|c|}
\hline Nr. & Vorgang & $01 / 00$ & $02 / 00$ & $03 / 00$ & $04 / 00$ & $05 / 00$ & & $6 / 00$ & $07 / 00$ & & $08 / 0$ & & $09 / 00$ & $10 / 00$ \\
\hline 1 & Kick-Off & $\square$ & & & & & & & & & & & & \\
\hline 2 & Prozessvision & & $\mathrm{HMa}$ & LSc, LBu & & & & & & & & & & \\
\hline 3 & Leistungsanalyse & & $\square$ & $\mathrm{HMa}, \mathrm{LSc}$, & MRi, LBu & & & & & & & & & \\
\hline 4 & Ablaufplanung & & & & a, LSc & & & & & & & & & \\
\hline 5 & Mikro-Prozess-Planung & & & & $\mathrm{HMa}, \mathrm{L}$ & $\mathrm{Sc}, \mathrm{Mri}, \mathrm{L}$ & & & & & & & & \\
\hline 6 & Prozessführung & & & & $\mathrm{HM}$ & a, LSc, DE & & & & & & & & \\
\hline 7 & Phasenabschlussbericht & & & & $\mathrm{HMa}, \mathrm{L}$ & & & & & & & & & \\
\hline 8 & Präsentation Phasenabschluss & & & 31.03. & & & & & & & & & & \\
\hline 9 & IS-Planung & & & & & & & & & & & & & \\
\hline 10 & Anwendungsentwurf & & & & L & 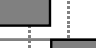 & & & & & & & & \\
\hline 11 & Implementierung & & & & & & & & & & & & & \\
\hline 12 & Abnahmetest & & & & & & & & & & & & & \\
\hline 13 & Schulung & & & & & & & & & & & & & \\
\hline 14 & Einführung & & & & & & & & & & & & & 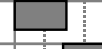 \\
\hline 15 & Inbetriebnahme & & & & & & & & & & & & & L \\
\hline 16 & Projektabschluss & & & & & & & & 1 & & & & & 30.10 . \\
\hline
\end{tabular}

Abb. 20. Projektzeitplan

Eine vollständigere Darstellung der Ergebnisse des Business Engineering findet sich in [Blessing/Österle 1999]. 


\section{Grundsätze des Business Engineering}

\section{Ziele des Business Engineering}

Das Business Engineering zerlegt also die Transformation in Projekte (im Projektportfolio), ein Projekt in den fachlichen Entwurf und in die Führung der Veränderung. Den fachlichen Entwurf wiederum zerlegt das Business Engineering in die Ebenen Strategie, Prozess und System. So entstehen kleine, beherrschbare Projektaktivitäten mit klar definierten Ergebnissen in Form von Dokumenten. Ein Vorgehensmodell schliesslich verbindet die Aktivitäten wieder zu einer Reihenfolge im Projektplan.

Das Business Engineering verfolgt damit das Ziel, die Transformation schnell und sicher zu machen sowie den Aufwand zu begrenzen. Es macht die Transformation führbar.

\section{Method Engineering}

Um die nötige Stringenz in den Projekten zu erzielen, befolgen wir die Grundsätze des Method Engineering [vgl. Gutzwiller 1994, S. 11 ff.]:

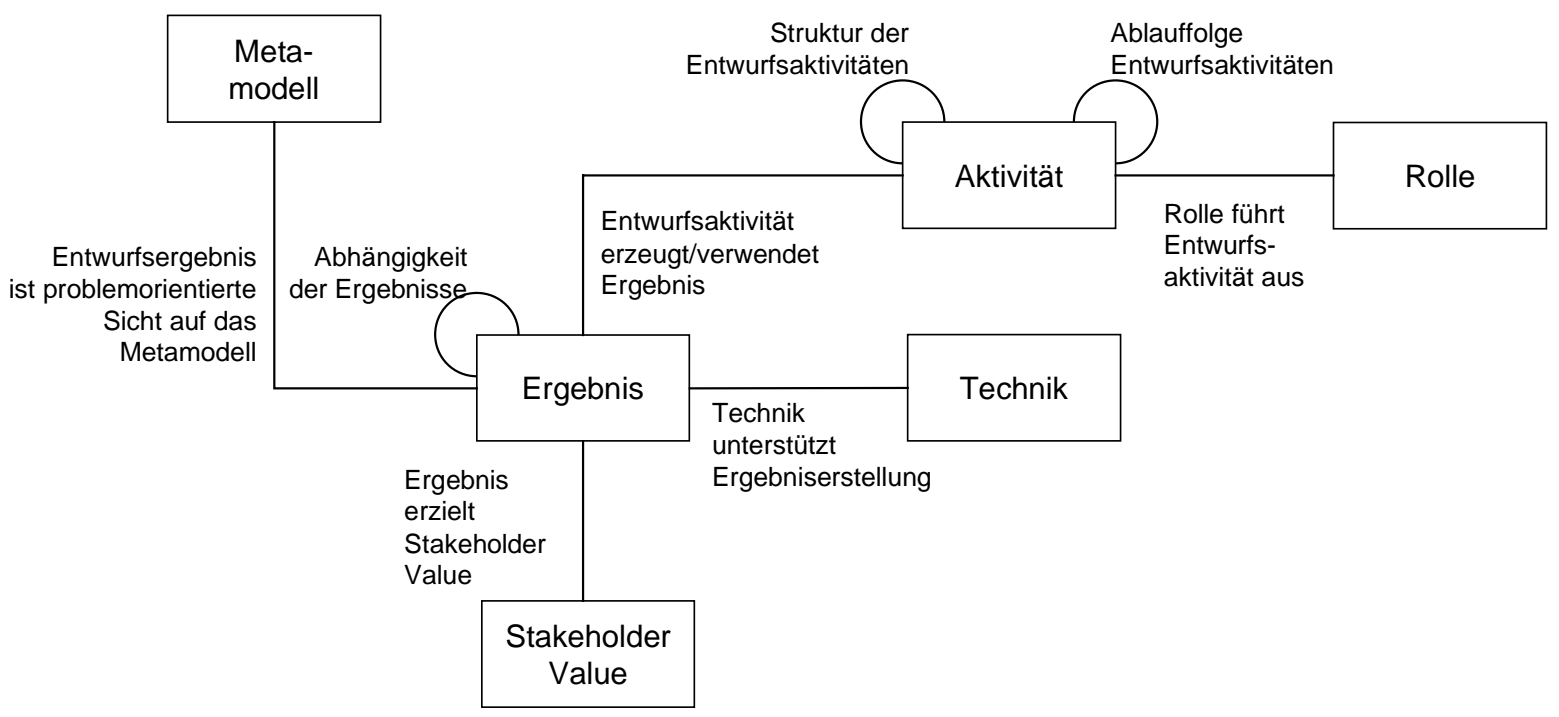

Abb. 21. Komponenten von Methoden

\section{- Ergebnisse}

Business Engineering ist ergebnisorientiert. Die reine Durchführung von Aktivitäten sichert noch keinen Erfolg. Jede Projektaktivität muss zu definierten Ergebnissen (meist Dokumente) führen, wie sie am Beispiel der Intertainment.com gezeigt wurden.

- Techniken

Die Techniken leiten den Business Engineer bei der Erarbeitung der Ergebnisse an. Sie fokussieren auf die kritischen Fragestellungen.

- $\quad$ Aktivitäten

Ein Projekt besteht aus einer Folge von Aktivitäten (Vorgehensmodell), die im Projektplan bzw. in Aktivitätenplänen festgelegt werden. Die Techniken helfen bei der Ausführung der Aktivitäten.

- Stakeholder Value

Das Kriterium für jede Geschäftslösung ist der Stakeholder Value. Auf der Strategie-Ebene legt die Geschäftslogik die Erfolgsfaktoren fest, die auf allen Ebenen zur Messung des Projekt- und Geschäftserfolges heranzuziehen sind. Ein wichtiger Teil daraus ist die Wirtschaftlichkeitsbetrachtung, beispielsweise in Form des Return on Investment (ROI).

- Rollen

Die Mitwirkenden in einem Projekt nehmen bestimmte Rollen wahr, die mit entsprechenden Aufgaben, Kompetenzen und Verantwortung verbunden sein müssen. Es sind dies beispielsweise der Organisator, der Programmierer, der Projektleiter, der Anwender und der Projektsponsor. 


\section{Metamodell}

Das Metamodell ist das Datenmodell des Business Engineering. Es beschreibt die einzelnen Gestaltungsobjekte (z. B. Prozess, Aufgabe, Kunde, Applikation) und die Beziehungen zwischen diesen. Abb. 22 zeigt einige wichtige Objekte des Business Engineering und ihre Zusammenhänge [vgl. zur Metamodellierung Ferstl/Sinz 1998, S. 117 ff., Scheer 1998].

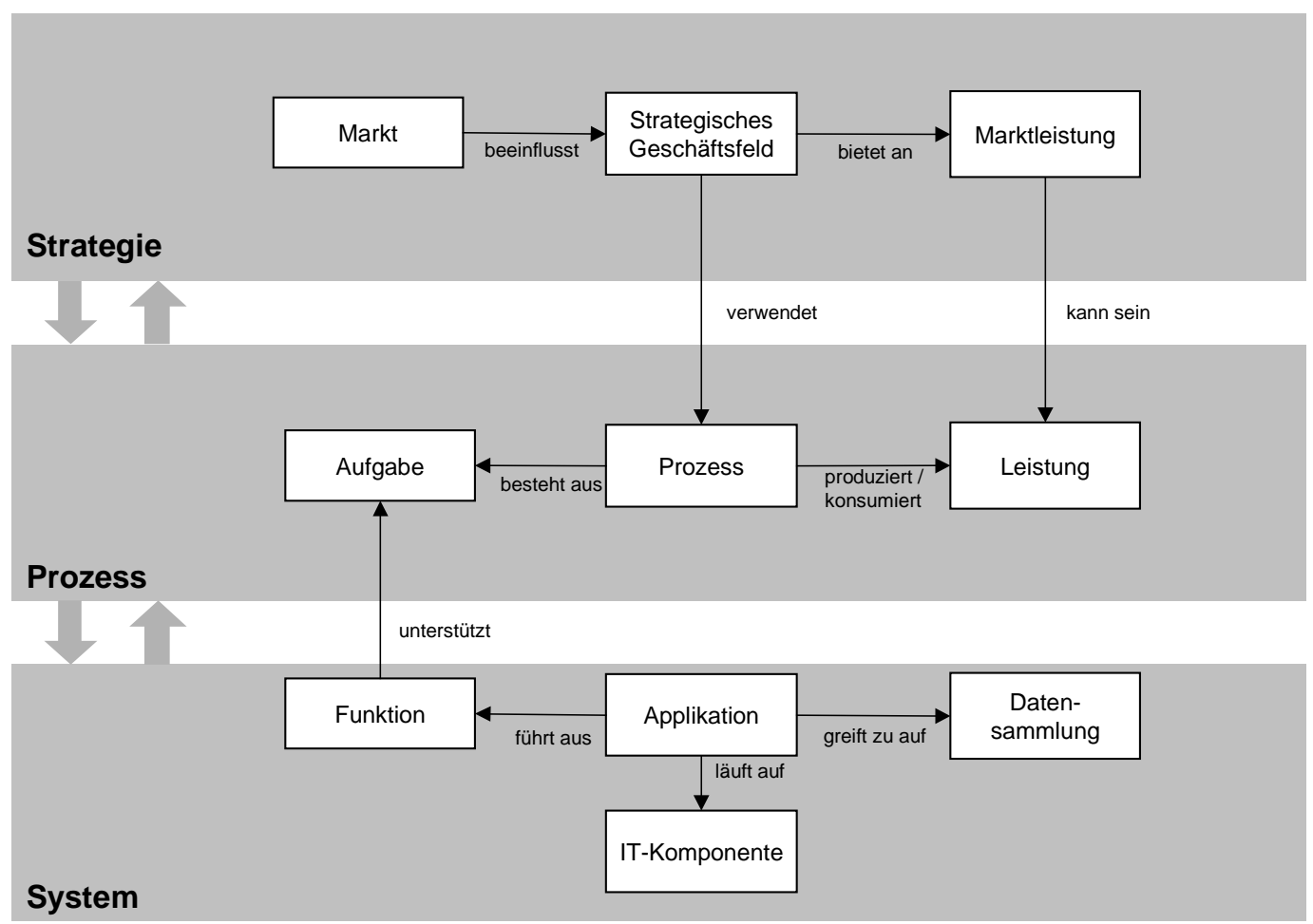

Abb. 22. Metamodell des Business Engineering

\section{Weitere Prinzipien des Business Engineering}

- Business Engineering umfasst den fachlichen Entwurf und das Change Management

Die fachliche Geschäftslösung ist nur ein Teil des Projektergebnisses. Diese muss schliesslich von den Betroffenen verstanden, gewollt und bewältigt werden. Kulturelle Faktoren sind daher ebenso wie politische einzubeziehen.

- $\quad$ Business Engineering trennt die Gestaltungsebenen des Unternehmens.

Business Engineering unterscheidet die Gestaltungsebenen Strategie, Prozess und System. Spielt die Informationstechnik in einem Projekt eine dominante Rolle, so kann zur Komplexitätsbeherrschung die Ebene System in die Ebenen Informationssystem und Informationstechnik aufgeteilt werden.

- Business Engineering sorgt für eine ganzheitliche Sicht über alle Dimensionen.

Business Engineering entwirft nicht nur Geschäftslösungen, sondern setzt diese bis zum Betrieb der Prozesse und Systeme um. Es muss sich daher um alle Dimensionen (betroffene Ressourcen und Prozesse) kümmern (vgl. Abb. 23), auch wenn wir im Beispiel auf die Informationsverarbeitung fokussiert haben. 


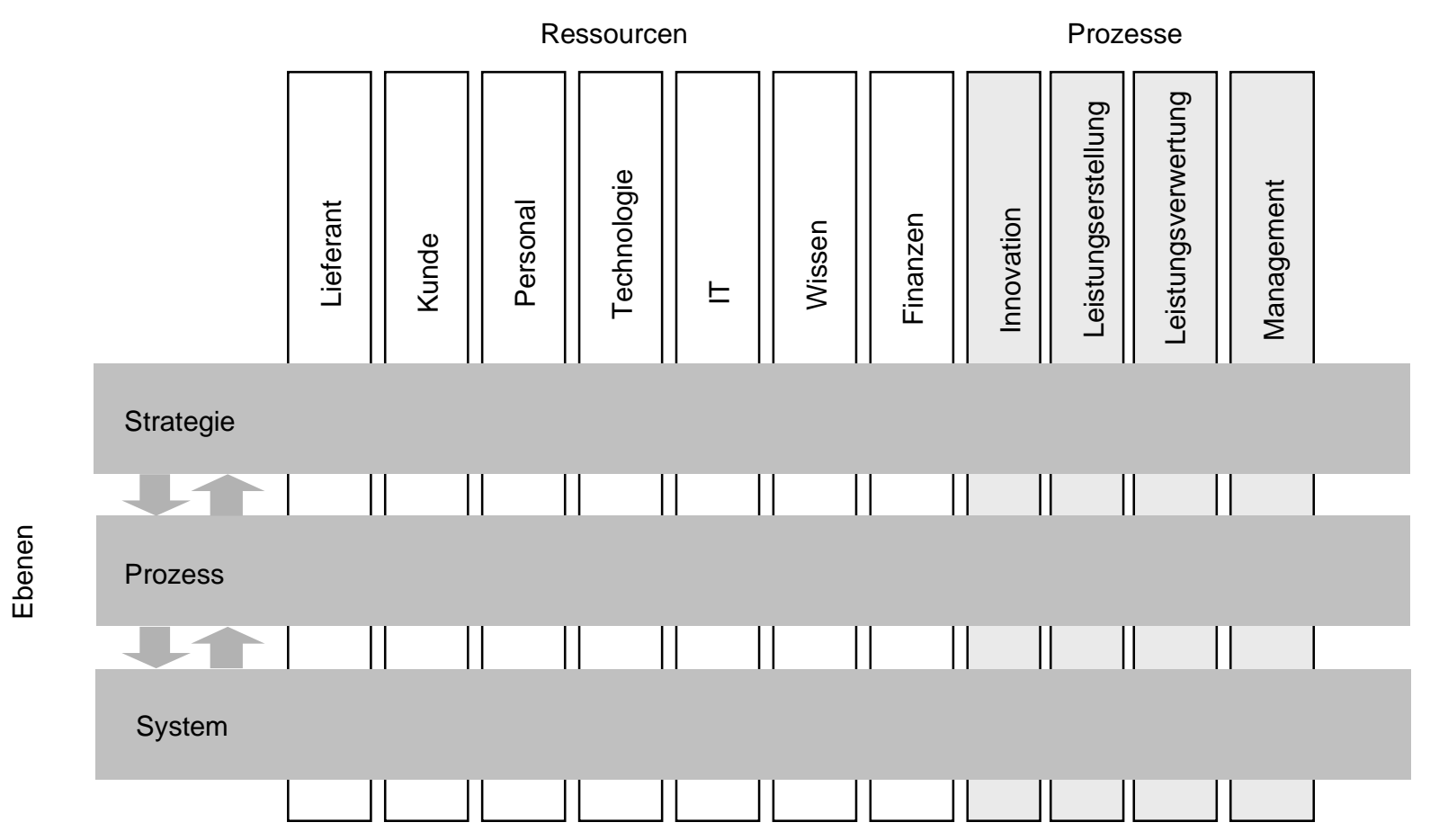

Abb. 23. Ebenen und Dimensionen des Business Engineering

\section{Methoden und Werkzeuge für das Business Engineering}

\section{Business Engineering am Beispiel von PROMET}

PROMET ist eine Familie von Methoden. Diese sind entweder auf bestimmte Bereiche (z. B. Strategie, Workflow-Management oder Projektmanagement) eines Projektes oder aber auf bestimmte Typen von Projekten spezialisiert (z. B. Customer Relationship oder Knowledge Management). Das Institut für Wirtschaftsinformatik der Universität St. Gallen hat in den letzten zehn Jahren zusammen mit führenden Unternehmen eine Reihe von Methoden entwickelt. Die IMG AG hat diese Methoden übernommen, weiterentwickelt und um weitere Methoden ergänzt. Heute steht mit PROMET ein Methodenset zur Verfügung, das sich in einer grösseren Zahl von Unternehmen seit Jahren bewährt hat [vgl. z. B. IMG 1997a, IMG 1997b, IMG 1998, www.img.com].

Die besonders relevanten Techniken und Dokumente sind im PROMET Kern zusammengefasst. (vgl. Abb. 1). Dieser stellt das Rückgrat jedes Transformationsprojektes dar. Für spezifische Problemstellungen existieren Erweiterungen zum Kern. Diese bestehen aus einem eigenständigen Vorgehensmodell sowie zusätzlichen Ergebnisdokumenten, Techniken und Rollen.

\section{Werkzeugunterstützung}

Für das Management von Business Engineering-Wissen stehen mittlerweile einige Spezialtools zum Methoden- und Projektmanagement zur Verfügung. Folgende Realisierungsalternativen bieten sich an:

Modellierung von Geschäftsprozessen

Im Mittelpunkt dieser Kategorie steht die Darstellung der Aufbau- und Ablauforganisation in Form von Diagrammen sowie die Dokumentation und Publikation der Ergebnisse. Die hinterlegte Methode fokussiert meist auf die graphische Darstellung; die Tools sind nur beschränkt offen für andere Methoden und beinhalten selten Vorgehensmodelle. Wichtige Vertreter sind ARIS Toolset von IDS-Scheer (www.ids-scheer.de) und Bonapart von proubis (www.proubis.de).

Projektmanagement

Schwerpunkt von Projektmanagement-Tools ist die Planung und Steuerung der finanziellen und personellen Projektressourcen sowie das Berichtswesen [vgl. auch Patzak/Rattay 1998, S. 575 ff.]. Methoden für 
bestimmte Problemstellungen sind in den Tools nicht verfügbar. Bekannte Produkte dieser Kategorie sind Project von Microsoft (www.microsoft.com) oder SuperProject/Net von Computer Associates (www.cai.com).

\section{Methodenunterstützung}

Vereinzelt stehen für bestimmte Fragestellungen spezielle Tools zur Verfügung. So bietet die SAP (www.sap.com) für die Einführung der ERP-Software R/3 AcceleratedSAP (ASAP) an, das neben dem Projektmanagement ein Vorgehensmodell, Techniken (Handbücher, Fragebögen, Checklisten) sowie Ergebnisdokumente (Templates) enthält. Für bestimmte Themen, wie Upgrade-Projekte, globaler Roll-out oder die Einführung von speziellen Produkten stehen zwischenzeitlich spezielle ASAP-Versionen zur Verfügung [vgl. SAP 1999].

Projektteamwork

Die Zusammenarbeit innerhalb von Projekten wird teilweise durch spezielle Module von Dokumentenmanagement- oder Groupwaresystemen unterstützt. Dabei können Aktivitäten, Projektergebnisse, Referenzen und Mitarbeiter verwaltet und für unterschiedliche Rollen (Projektmanager, Projektmitarbeiter) dargestellt werden. Teilweise wird auch die Verwendung von vordefinierten Methoden unterstützt. Beispiele aus diesem Bereich sind iTeam von Documentum (www.documentum.com) oder Livelink von Opentext (www.opentext.com).

\section{Zusammenfassung und Ausblick}

Business Engineering ist die Konstruktionslehre für den Organisator des Informationszeitalters. Einige Themen wie etwa der Entwurf von Prozessen sind bereits in vielen Projekten erprobt und damit ausgereift, andere Gebiete wie etwa das Change Management sind komplexer und schwerer strukturierbar. In einer Idealvorstellung benutzt der Business Engineer der Zukunft ein spezifisches ProzessPortal, das ihm alles Wissen, das er für die Transformation benötigt, verwendungsgerecht anbietet. Einen Teil davon wie etwa die Grundlagen des Business Engineering wird er in Form von Ausbildung abrufen, einen Teil wie beispielsweise ein Vorgehensmodell zur Einführung eines bestimmten Standardsoftwarepaketes wird er im Bedarfsfall als Vorlage abrufen und sich erklären lassen. Die Wissenschaft (z. B. brint.com), die Softwarehäuser (z. B. sap.com), die Beratungshäuser (z. B. ac.com), Communities (z. B. businesstech.com oder cio.com) und viele weitere werden die Inhalte für dieses Portal liefern.

Ein grosses Problem auf dem Weg zum umfassenden BusinessEngineeringPortal ist die Heterogenität dieses Wissensbereiches und die mit dem Volumen verbundene Komplexität. Zudem sind heute gerade mühsam erarbeitete Strukturen z. B. zur Funktionalität von Customer Relationship Management Paketen aufgrund der rasanten Innovation in diesen Bereichen morgen möglicherweise bereits überholt. Daher müssen wir damit rechnen, dass mögliche Formen von BusinessEngineeringPortalen bis auf weiteres noch recht rudimentär sein müssen.

\section{Literatur}

[Aschwanden 1998]

Aschwanden, C., IT Governance in Banken, Tagungsband der 4. Handelsblatt-Jahrestagung Banken-Technologie 98/99, Frankfurt a. M., 1998

[Blessing/Österle 1999]

Blessing, D., Österle, H., Business Engineering Model, Arbeitsbericht des Instituts für Wirtschaftsinformatik der Universität St. Gallen, St. Gallen, 1999

[Dolmetsch et al. 1998]

Dolmetsch, R., Huber, T., Fleisch, E., Österle, H., Accelerated SAP, 4 Case Studies, Arbeitsbericht des Instituts für Wirtschaftsinformatik der Universität St. Gallen, St. Gallen, 1998

[Ferstl/Sinz 1998]

Ferstl., O. K., Sinz, E., E., Grundlagen der Wirtschaftsinformatik, Band 1, 3. Aufl., Oldenbourg, München/Wien, 1998 
[Gomez/Probst 1991]

Gomez, P., Probst, G., Die Praxis des ganzheitlichen Problemlösens, Vernetzt denken -

Unternehmerisch handeln - Persönlich überzeugen, 2. Aufl., Haupt, Bern u. a., 1997

[Gutzwiller 1994]

Gutzwiller, T., Das CC RIM-Referenzmodell für den Entwurf von betrieblichen, transaktionsorientierten Informationssystemen, Physica, Heidelberg, 1994

[IMG 1997a]

Information Management Gesellschaft, PROMET®-BPR - Methodenhandbuch für den Entwurf von Geschäftsprozessen, Version 2.0, St. Gallen et al., 1997

[IMG 1997b]

Information Management Gesellschaft, PROMET®-SSW - Methodenhandbuch für die Einführung von Standardanwendungssoftware, Version 3.0e, St. Gallen u. a., 1997

[IMG 1998]

Information Management Gesellschaft, PROMET®-INET - Methodenhandbuch für die Planung und Konzeption von Intranets, Version 1.01, St. Gallen u. a., 1998

[Kunii 1999]

Kunii, I., Here Comes the Sony Netman, The electronics giant thinks its future is as a player on the web, Business Week, 1. November 1999, S. 28 - 29

[Patzak/Rattay 1998]

Patzak, G., Rattay, G., Projekt-Management, 3. Aufl., Linde Verlag, Wien, 1998

[SAP 1999]

SAP AG, ASAP - die Methode zur schnellen R/3 Einführung, Walldorf, http://www.sapag.de/germany/products/imple/asap/index.htm (22.11.1999)

[Scheer 1998]

Scheer, A.-W., ARIS - Vom Geschäftsprozess zum Anwendungssystem, 3. Aufl., Springer, Berlin u. a., 1998 